\title{
The GIP/GIPR axis is functionally linked to GH-secretion increase in a significant proportion of gsp- somatotropinomas
}

\author{
D Regazzo', M Losa², N M Albiger', M R Terreni³ ${ }^{3}$ G Vazza4, F Ceccato', E Emanuelli5, \\ L Denaro ${ }^{6}$, C Scaroni ${ }^{1}$ and $\mathbf{G ~ O C c h i ~}^{4}$ \\ ${ }^{1}$ Endocrinology Division, Department of Medicine, Hospital/University of Padova, Padova, Italy, \\ 2Pituitary Unit, Department of Neurosurgery, ${ }^{3}$ Pathology Unit, Istituto Scientifico San Raffaele, \\ Università Vita-Salute, Milano, Italy, ${ }^{4}$ Department of Biology, University of Padova, Padova, Italy, \\ ${ }^{5}$ Otolaryngology and Otosurgery Unit, and ${ }^{6}$ Department of Neuroscience, Hospital/University of Padova, \\ Padova, Italy
}

\author{
Correspondence \\ should be addressed \\ to G Occhi \\ Email \\ gianluca.occhi@unipd.it
}

\begin{abstract}
Objective: Glucose-dependent insulinotropic polypeptide receptor (GIPR) overexpression has been recently described in a proportion of $\mathrm{gsp}^{-}$somatotropinomas and suggested to be associated with the paradoxical increase of GH (GH-PI) during an oral glucose load.

Design and methods: This study was aimed at linking the GIP/GIPR pathway to GH secretion in 25 somatotropinomasderived primary cultures and correlating molecular with clinical features in acromegalic patients. Given the impairment of the GIP/GIPR axis in acromegaly, an additional aim was to assess the effect of GH/IGF-1 stimulation on GIP expression in the enteroendocrine cell line STC-1.

Results: Nearly $80 \%$ of GIPR-expressing somatotropinomas, all of them negative for gsp mutations, show increased GH secretion upon GIP stimulation, higher sensitivity to Forskolin but not to somatostatin analogs. Besides increased frequency of GH-PI, GIPR overexpression does not appear to affect acromegalic patients' clinical features. In STC-1 cells transfected with GIP promoter-driven luciferase vector, IGF-1 but not GH induced dose-dependent increase in luciferase activity.

Conclusions: We demonstrate that GIPR mediates the GH-PI in a significant proportion of $g s p^{-}$acromegalic patients. In these cases, the stimulatory effect of IGF-1 on GIP promoter support the hypothesis of a functional GH/IGF-1/GIP axis. Further studies based on larger cohorts and the development of a stable transgenic model with inducible GIPR overexpression targeted to pituitary somatotroph lineage will be mandatory to establish the real role of GIPR in the pathogenesis of somatotropinomas.
\end{abstract}

\section{Introduction}

Acromegaly is a rare and progressive hormonal syndrome commonly due to a benign monoclonal GH-secreting pituitary adenoma (GH-sec PA) (1). Patients often have well-known systemic complications at diagnosis with increased morbidity and mortality (2) and reflecting the
European Journal of Endocrinology

(2017) 176, 543-553
() 2017 European Society of Endocrinology Printed in Great Britain slow progression of the symptoms over a period of many years, and the clinical diagnosis is frequently delayed.

A biochemical diagnosis of acromegaly is made by quantifying serum IGF-1 levels and determining autonomous GH secretion by measuring its levels after 
a standard oral glucose challenge (OGTT). In patients with active disease, oral glucose fails to suppress serum GH levels to below $0.4 \mu \mathrm{g} / \mathrm{L}$, and in approximately $30 \%$ of cases, it increases paradoxically (1).

With the aim of clarifying the pathogenesis of GH-secreting PAs, we recently focused our attention on a member of the G-protein-coupled receptors superfamily (GPCR) - i.e. the glucose-dependent insulinotropic polypeptide receptor (GIPR) (3). Once activated by GIP, which is secreted by the $\mathrm{K}$ cells of the duodenum in response to meals, GIPR transduces extracellular stimuli into intracellular responses by activating the cAMP pathway $(4,5)$. In physiological conditions, the glucosedependent secretion of insulin in pancreatic $\beta$ cells after GIP stimulation exemplifies this mechanism (6). When inappropriately expressed in the adrenal gland, the GIPR may instead result in the development of an adrenal tumor (7), disrupting cAMP homeostasis by altering the cascade normally triggered by ACTH. Consequently, this leads to increased cortisol secretion and the development of food-dependent Cushing's syndrome (FDCS) $(8,9)$.

We found that nearly $30 \%$ of GH-sec PA expressed GIPR at significantly higher levels than normal pituitary glands. All were $g s p$ negative, thus suggesting that these two events might be mutually exclusive and perhaps parts of the same pathogenic mechanism involving cAMP (3). Correlation of molecular findings with clinical data revealed that in most cases, GIPR overexpression was associated with a paradoxical increase in GH after OGTT (GH-PI) $(3,10)$. Similar to what is observed in FDCS, in GIPR-overexpressing GH-sec PAs, GH is inducible by meal (10), but unlike FDCS in which cortisol levels drop in parallel with GIP reduction; in acromegalic patients, the GH blood concentrations always remain above the diagnostic threshold. Although such observation might suggest that GIPR may not be the primary cause of acromegaly (10), these patients show abnormally high fasting and postprandial plasma GIP levels (11); this may be the consequence of a direct effect of GH, IGF-1 or both on GIP secretion.

Based on these premises, we aimed to study if a link between the GIP/GIPR axis and GH induction in GH-secreting PAs primary cultures exists and to GH-PI observed in acromegalic patients. Secondly, taking advantage of the GIP-secreting enteroendocrine model cell line STC-1, we assessed the effect of GH/IGF-1 stimulation on GIP secretion. Clinical-to-molecular correlation among patients from the current and our previous series (3) is also reported.

\section{Subjects and methods}

\section{Patient tissues and primary cell cultures}

We studied 25 consecutive active acromegalic patients (15 females; age 17-70 years, mean age $44 \pm 15$ years) diagnosed at the Endocrinology Unit of Padova University/Hospital and at the Pituitary Unit, Department of Neurosurgery, San Raffaele Hospital in Milan on the basis of consensus criteria (12). OGTT was performed in all 25 patients. An arbitrary threshold of greater than $20 \%$ of GH increase during this test - to discriminate between real increase and test variability (see below) was set to differentiate paradoxical from non-paradoxical responders. Patients' clinical and hormonal features are reported in Supplementary Table 1 (see section on supplementary data given at the end of this article).

All 25 acromegalic patients underwent transsphenoidal surgery. Portions of the surgically removed specimens were fixed in 10\% buffered formalin and then embedded in paraffin; standard sections stained with hematoxylin and eosin were used for diagnosis, whereas the presence of pituitary hormones was evaluated by standard immunocytochemical analyses. A second fragment for each tissue specimen was immersed in RNAlater (Ambion), kept at $4^{\circ} \mathrm{C}$ for $24 \mathrm{~h}$ and then stored at $-20^{\circ} \mathrm{C}$ until RNA extraction. The remainder of each somatotropinoma was transferred to sterile cold complete culture medium and processed within $36 \mathrm{~h}$ as previously described (13). Informed consent was obtained from each patient. The study was conducted in accordance with the Helsinki Declaration.

\section{Primary cells treatments and GH measurement}

The effect of GIP and somatostatin analogs (SA) on GH secretion was examined in somatotropinoma-derived primary cells. Novartis Pharma AG kindly provided the SA Pasireotide (SOM230) and Octreotide (OCT), whereas GIP and Forskolin (FK) were purchased from Sigma-Aldrich.

After seeding and incubation of primary cells at $37^{\circ} \mathrm{C}$ for $48-60 \mathrm{~h}$, the medium was removed and replaced with $2 \%$ FBS DMEM containing GIP (100 nM), FK $(10 \mu \mathrm{M})$, OCT $(100 \mathrm{nM})$ or SOM230 $(100 \mathrm{nM})$. Cells were incubated for further $6 \mathrm{~h}$ (GIP and FK) or $24 \mathrm{~h}$ (OCT and SOM230) before the medium was recovered and frozen. GH was measured by Immulite 2000 (DPC, Carpinteria, CA, USA) (coefficient of variation (CV) $<7 \%$, lower detection limits $0.01 \mu \mathrm{g} / \mathrm{L})$. 


\section{Immunofluorescence}

Immunofluorescence for GIPR expression was performed on GH-sec PAs on conventional sections after deparaffinization in xylene, rehydration through graded alcohols to water and antigen retrieval (Dako) in $10 \mathrm{mM}$ sodium citrate buffer ( $\mathrm{pH} \mathrm{6.0)}$ for $10 \mathrm{~min}$ at $96^{\circ} \mathrm{C}$. Sections of a normal human pancreas and of a GH-sec PA incubated with non-immune serum were used as positive and negative controls respectively.

GIPR expression was visualized with a rabbit polyclonal antibody (kindly provided by Prof. Timothy Kieffer, University of British Columbia, Vancouver, Canada; O/N, $\left.4^{\circ} \mathrm{C}, 1: 250\right)$ and Alexa Fluor 594-labeled donkey antirabbit IgG secondary antibody (Life Technologies, 1:250). Tissue sections were also stained for $\mathrm{GH}$ by incubating with a mouse anti-GH monoclonal antibody (Santa Cruz Biotechnology, 1:200) and Alexa Fluor 488-labeled donkey anti-mouse IgG secondary antibody (Life Technologies, 1:250). Cell nuclei were stained with $1.5 \mu \mathrm{g} / \mathrm{mL}$ Hoechst 33258 (Sigma-Aldrich). The cover slips were rinsed twice in excess PBS and mounted with Fluorescent Mounting Medium (Dako, Cat. No. S3023). The preparations were examined with a DMI6000CS fluorescence microscope (Leica Microsystems CMS) with a 63×/1.40 oil-immersion objective. Images were acquired by means of a DFC365FX camera and analyzed with Leica LAS-AF 3.1.0 software.

\section{Nucleic acid isolation, cDNA synthesis and mutation screening}

The tumor specimens collected in RNAlater were homogenized in a TissueLyser (Qiagen) in $1 \mathrm{~mL}$ of TRIzol reagent (Invitrogen) using a modified TRIzol protocol (14). RNA and DNA yields were determined on a NanoDrop spectrophotometer (NanoDrop Technologies, Wilmington, DE, USA), and RNA integrity was tested with the Agilent 2100 Bioanalyzer (Agilent Technologies). Genomic DNA in the RNA was removed by DNAse, treating total RNA with Turbo DNA free kit (Ambion). RNA (500ng) was reverse-transcribed with M-MuLV Reverse Transcriptase RNase H- (Euroclone, Pero, Italy) according to the manufacturer's recommendations.

Somatotroph adenomas were screened for somatic gsp mutations as reported previously (3). In nine GIPR overexpressing cases - included in this and previous cohort (3) - of which we had both somatic and matched germline DNA, the presence of somatic mutations in GIPR promoter (15) and transcribed regions has been evaluated. Lymphocytes DNA was extracted with a DNeasy Blood and Tissue Kit (Qiagen) according to the manufacturer's instructions. DNA sequencing was performed using the BigDye 1.1 Termination Chemistry on an ABI 3730XL (Applied Biosystems). All primer sequences and PCR conditions are available upon request.

\section{Quantitative RT-PCR (RT-qPCR)}

RT-qPCR experiments were performed according to the MIQE guidelines. The GoTaq Probe qPCR Master Mix (Promega) and TaqMan Gene Expression Assays for the GIPR (Hs00609210_m1, Life Technologies) were used in an ABI PRISM 7900HT Sequence Detector (Applied Biosystems). GIPR flanking genes GPR4 (NM_005282; For 5'-agggaacataagaccgcaat-3'; Rev 5'-tgcccttcacttgagttctg-3'), EML2 (isoforms NM_001193268, NM_001193269, NM_012155 and NR_034098; For 5'-atgctgtcatccacaagaa-3'; Rev 5'-ttggcaaagttcacctgttt-3'), SNRPD2 (NM_004597; For 5'-ggagtgaacggagagcgtag-3'; Rev 5'-tcttattgttgcggcagttg-3') and QPCTL (isoforms NM_001163377 and NM_017659; For 5' -acctgctgcagtctcatcc-3'; Rev 5'-tgagatggagcacgggta-3') were assessed with the GoTaq qPCR Master Mix (Promega). Similarly, to assess the possible duplication of the GIPR-containing locus, two different genomic regions (CNV1, AC006132 For 5'-gtggcgggttgtgatgga-3', Rev 5'-ccctcccttgccgattg-3'; CNV2, AC007191 For 5'-cgggtagggggagaacca-3', Rev 5'-ctcgccctctcctgaga-3') were assessed by qPCR as previously described (16). For this purpose, tumoral DNA was extracted with a DNeasy Blood and Tissue Kit (see previously). A final concentration of either 300nM (GPR4, EML2, CNV1 and CNV2) or $50 \mathrm{nM}$ (SNRPD2 and QPCTL) for both forward and reverse primers was used. All samples were tested in duplicate in a MicroAmp 96-well reaction plate sealed with an optical adhesive film (Applied Biosystems) with a proper amount of template (5-20ng of cDNA or $10 \mathrm{ng}$ gDNA) in $20 \mu \mathrm{L}$ of reaction mixture. For GIPR, the reaction was set up using $20 \mathrm{ng}$ of template. No-template controls were included in each run. The PCR conditions were $95^{\circ} \mathrm{C}$ for $2 \mathrm{~min}$, followed by 40 cycles at $95^{\circ} \mathrm{C}$ for $15 \mathrm{~s}$ and at $60^{\circ} \mathrm{C}$ for $1 \mathrm{~min}$. Data were analyzed with SDS rel.2.4 (Applied Biosystems), with an automatically set baseline and a fluorescence threshold adjusted for measuring quantification cycle (Cq) values. Validation experiments performed using the standard curve method with five serial dilutions of genomic DNA from control subjects showed identical amplification efficiencies $(100 \% \pm 10 \%)$ calculated according to: $E=10^{1 / \text {-slope }}-1$, for all assays. The amount of each target gene relative to 
pituitary adenomas' most stable housekeeping gene, HMBS (14) for RT-qPCR and to a reference locus on chromosome 19 (GPR4 gene, see above) for qPCR was ascertained by the $\Delta \Delta \mathrm{Cq}$ method. The threshold for distinguishing high(GIPR-H) and low-expressing GIPR (GIPR-L) specimens was established previously (3).

\section{Cell lines, transfection and dual-luciferase assay}

The possible interaction between the GH/IGF-1 axis and the synthesis and/or secretion of GIP was investigated with murine enteroendocrine cell lines STC-1 (ATCC CRL-3254). Cells were cultured at $37^{\circ} \mathrm{C}$ and $5 \% \mathrm{CO}_{2}$ in DMEM-low glucose (ECM0749, Euroclone) supplemented with $10 \%$ FBS, $3.7 \mathrm{~g} / \mathrm{L} \mathrm{NaHCO}_{3}, 2$ mM L-glutamine, $100 \mathrm{U} /$ $\mathrm{mL}$ penicillin and $100 \mathrm{mg} / \mathrm{mL}$ streptomycin. Twenty-four hours before the experiment, STC- 1 cells $\left(1.75 \times 10^{5}\right.$ cells/ well) were seeded into 12 -well plates. Cells were transiently transfected with $2 \mu \mathrm{L}$ of Lipofectamine 2000 (Invitrogen) together with $1.6 \mu \mathrm{g}$ of total DNA consisting of hGIP2.9kbluc (a kind gift from Prof. T Kieffer generated by cloning a 2.9-kb fragment of human GIP promoter (-2844 to $+57 \mathrm{bp})$ upstream luciferase gene (17)) and pRL-TK (Promega) and incubated for $24 \mathrm{~h}$. Cells were then treated for further $24 \mathrm{~h}$ with $\mathrm{GH}$ (from 1 to $100 \mathrm{ng} / \mathrm{mL}$ ), IGF-1 (from 0.1 to $100 \mathrm{ng}$ / $\mathrm{mL})$, insulin (100 $\mathrm{nM})$ or the combination of FK and IBMX $(10 \mu \mathrm{M}$ each) and the effect on GIP promoter activity was examined. All compounds used for cell treatments were purchased from Sigma-Aldrich.

Cell media were removed, proteins were harvested in passive lysis buffer (Promega) and the relative luciferase activity was measured with the Dual-Luciferase Reporter Assay System and a GloMax 20/20 luminometer (Promega), according to the manufacturer's instructions.

As part of the study, RT-PCR was used to detect the presence of $\mathrm{GH}(G H-R)$ and IGF-1 receptors $(I G F-1 R)$ in STC-1 cells. RNA was extracted and reverse-transcribed as reported previously. Twenty-five nanograms of cDNA was amplified for 40 cycles with Taq G2 Flexi DNA Polymerase (Promega) and 5 pmol of sense and antisense primers, common to all known isoforms ( $G H-R$, Primer F 5'-ggtcttcttaaccttggc-3', Primer R 5'-tcttgcagcttgtcgttg-3', $I G F-1 R$, Primer F $5^{\prime}$-atggagtgctgtatgcttctg-3', Primer R $5^{\prime}$-cggttcatggtgatcttctctc-3'). Mouse liver cDNA was used as a positive control for $G H-R$ and $I G F-1 R$ expression.

\section{Statistical analysis}

For cell culture studies, all experiments were performed at least twice, and results are presented as the mean \pm S.D. of at least triplicate determinations. Significance was determined by Student's $t$-test. To correlate clinical with molecular and biochemical data proportions and rates for categorical variables, means and standard deviations or medians and inter-quartile ranges (IQR) for parametric or non-parametric variables were calculated. Groups were compared with the chi-square test for categorical variables (or Fisher's exact test when the cell count was $<5$ ) or the Mann-Whitney test for quantitative variables, as appropriate. The SPSS 17 software package (SPSS) was used for all analyses. The significance level was set at $P<0.05$ for all tests.

\section{Results}

\section{Clinical and molecular analysis}

Tumor specimens have been evaluated for GIPR expression by RT-qPCR. Based on our previous observations and established criteria (3), samples were divided into two distinct subgroups. The first comprises 15 samples expressing GIPR within the range for normal pituitaries (GIPR-L; from $1.6 \times 10^{-4}$ to $1.7 \times 10^{-2}$, mean $\left.2.9 \times 10^{-3} \pm 4.6 \times 10^{-3}\right)$, and the second comprises 10 samples expressing GIPR at significantly higher levels (GIPR-H; from 0.02 to 0.27 , mean $0.13 \pm 0.08$ ) (Fig. 1). By immunofluorescence we confirmed the high GIPR expression in the adenoma sections in the latter cases, both membrane and cytoplasmic immunoreactivity (3). Co-localization of red and green staining in the same cell confirmed that GIPR is expressed in GH-secreting tumor cells (Fig. 2).

The thorough assessment of clinical features in the 25 acromegalic patients revealed that 8/10 GIPR-H patients showed a GH-PI (Fig. 3 and Supplementary Table 1). The remaining two patients showed a decrease of $\mathrm{GH}$ that did not fall below $0.4 \mu \mathrm{g} / \mathrm{L}$ (\#6) or a slight increase not exceeding the previously established cut-off of $120 \%$ (\#9). In contrast, only 3/15 GIPR-L patients (\#5, \#7 and \#15) showed a GH-PI. Among GIPR-H subjects with a postoperative OGTT, one was cured by adenomectomy and the paradoxical GH secretion was abolished. In three further cases, despite evidence of a markedly reduced nadir $\mathrm{GH}$ in two of them, persistence of GH levels above $0.4 \mu \mathrm{g} / \mathrm{L}$ confirmed the persistence of the diseases (Supplementary Table 1); the GH-PI was also maintained.

Seeking the molecular cause of GIPR gene overexpression, we studied the GIPR locus in GIPR-H tumors. On sequence analysis, no somatic mutations were identified. To evaluate a possible impairment of 


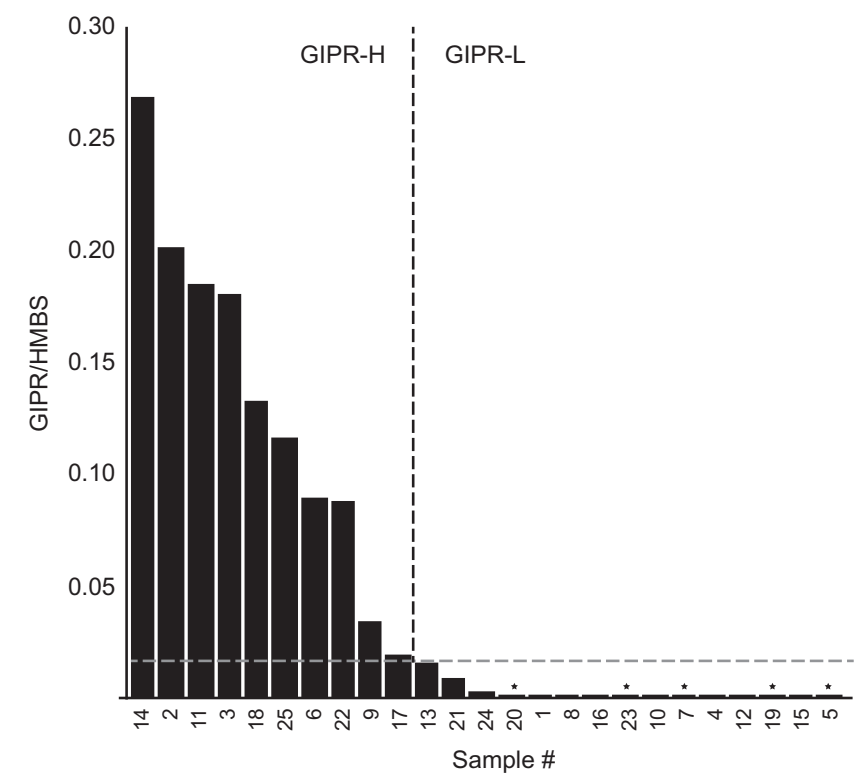

Figure 1

GIPR mRNA steady-state level in tumor tissue samples. GIPR mRNA levels were normalized against the most stable pituitary housekeeping gene, HMBS. Patients' identification numbers have been reported in Supplementary Table 1. The dashed gray line represents the threshold above which samples were classified as overexpressing GIPR (see 'Material and methods' section). The dashed black line divides the chart in two parts that include GIPR-H (left) and GIPR-L (right) samples. ${ }^{\S} g s p^{+}$.

transcription involving the 19q13.32 locus (SF 1a), we compared the steady-state level of GIPR's upstream (GPR4 and EML2) and downstream flanking genes (SNRPD2 and QPCTL) mRNA between six GIPR-H and ten GIPR-L samples. No significant difference emerged between the two groups (see SF 1b). In addition, copy number gains of GIPR were excluded by qPCR testing two different genomic regions (CNV1 and CNV2 in SF 1a) in two GIPR-H and five GIPR-L specimens respectively (SF 1c).

Tumor specimens were then further evaluated for gsp mutations. As shown in Fig. 1, one of two mutations was identified in 5 of the 25 cases, and only associated with low GIPR expression. In all but one case with an $\mathrm{A} / \mathrm{T}$ transition at codon 227 (Q227L), the mutation occurred at codon 201 (three R201C and one R201H).

\section{Primary cell treatment and GH measurement}

To definitively confirm the link between GIP/GIPR and the GH-PI, somatotropinoma-derived primary cultures have been established and cells were treated with GIP.
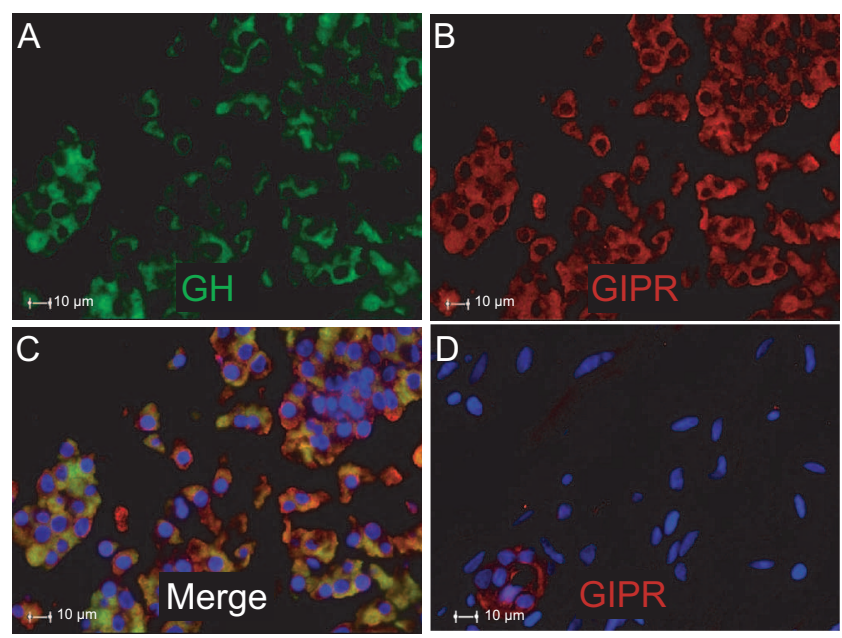

\section{Figure 2}

Representative immunofluorescence staining of a GH-sec PA with high GIPR expression. (A) GH is visualized in the green immunofluorescent channel, whereas (B) GIPR in the red one. (C) Co-localization of red and green staining in the same cell confirms that GH-secreting tumor cells express GIPR. Cells are counterstained with Hoechst (blue) to mark nuclei. (D) Positive control is a section of a normal human pancreas.

Immunofluorescent images are at 100x magnification.

As shown in Fig. 4A, 6/10 GIPR-H cases significantly responded to GIP stimulus (GH secretion mean induction $204 \% \pm 95 \%$ ). In the remaining four cases, two (\#6 and \#22) showed a GH increase - 121\% and 137\% respectively

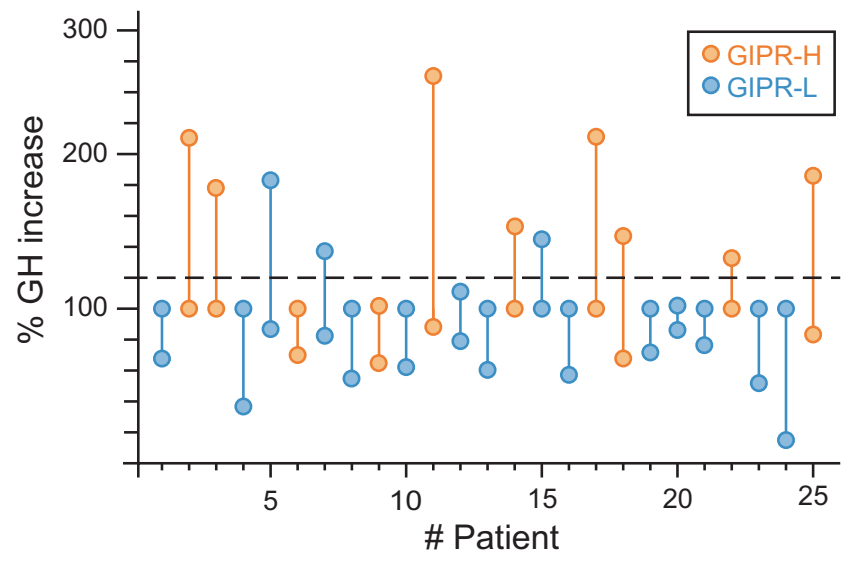

Figure 3

Zenith and nadir of two-hour OGTT in the cohort of acromegalic patients. Values are expressed as percentage of the zero time point. The dashed black line represents the $120 \%$ threshold we considered for defining an increase as paradoxical. 

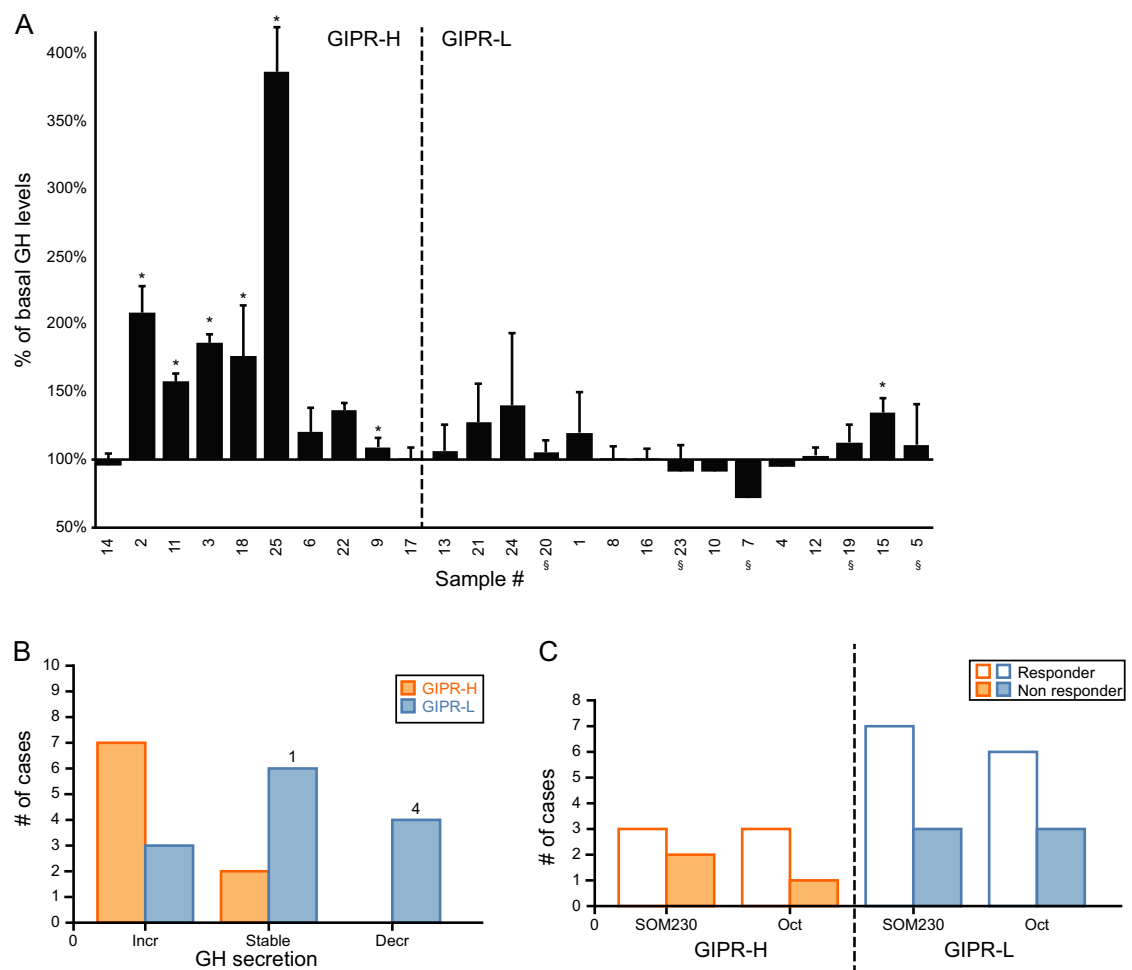

\section{Figure 4}

GH-sec PA-derived primary cultures responsiveness to different stimuli. (A) Cell cultures have been treated with $100 \mathrm{nM}$ GIP or vehicle and $\mathrm{GH}$ secretion has been evaluated. The level of $\mathrm{GH}$ secretion was expressed as relative percentage to vehicle-treated tumor cells. ${ }^{\S} g s p^{+},{ }^{\star} P<0.05$. Samples have been ordered according to Fig. 1 to easily compare GIPR expression data with functional in vitro studies. (B) Primary cultures responsiveness to FK $(10 \mu \mathrm{M})$. Bars represent the number of samples that either showed a statistically significant increase (incr), a decrease (decr) of GH after FK treatment. Stable are those cultures in which GH secretion is not influenced by FK treatment. The number of $g s p^{+}$cases in each group, when different from 0 , is reported above the correspondent bar. (C) Primary cultures responsiveness to SOM230 or Oct (100 nM) divided according to the GIPR expression profile. Responders are considered as those cases with a statistically significant decrease of GH secretion compared to the correspondent vehicle-treated cells.

- that did not reach statistical significance (due to high variability among vehicle-treated wells). In contrast, among GIPR-L primary cells, only in one case (\#15) a modest increase of GH secretion after GIP stimulation was observed. In vitro GH increase due to GIP treatment and GIPR overexpression were positively correlated (Spearman's Rho $R=0.522, P=0.006$ ).

To characterize the activation status of the cAMP signaling pathway, according to GIPR expression, primary adenoma cells were treated with FK and GH secretion was evaluated. As shown in Fig. 4B, GIPR-H tumors responded more frequently $(7 / 9$ vs $3 / 13, P<0.05)$ and with higher response to FK $(185 \% \pm 113 \%$ vs $110 \% \pm 63 \%, P=0.05)$ than GIPR-L adenomas. Interestingly, in four of five $g s p^{+}$ tumors, FK treatment induced a significant reduction of GH (mean decrease $44 \% \pm 10 \%$ ) possibly as a consequence of a non-inducible pathway (18).
Whenever possible, primary cultures were treated with either OCT or SOM230, the SSTR2- and SSTR5selective agonists respectively. Both molecules were equally effective in inducing GH decrease in both GIPR-H and GIPR-L somatotropinomas (Fig. 4C).

\section{Clinical-to-molecular phenotype}

To increase the power to identify possible association, we then combined data from this and from a previously published cohort (3). This resulted in 47 cases (15 males) for which both molecular and comprehensive clinical data were available. The GH-PI was observed in $43 \%$ (20/47) of acromegalic patients and is strictly associated with GIPR expression in somatotropinomas but not to gsp mutational status. The group of 20 patients with a GH-PI included 81\% (13/16) and 23\% (7/31) of GIPR-H 
A

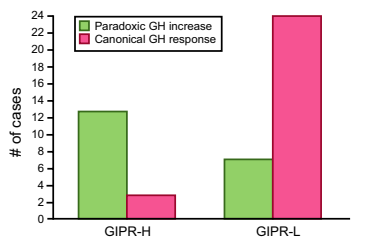

B

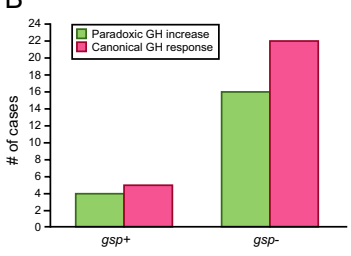

C

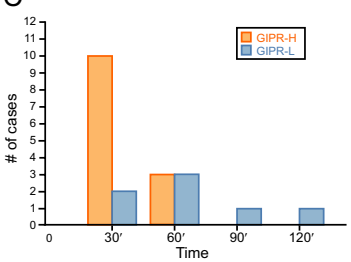

\section{Figure 5}

Correlation between biochemical and molecular observations in acromegalic patients. Subjects have been grouped according to (A) GIPR expression profile or (B) gsp mutational status. Bars represent the number of patients within each group presenting a paradoxical or a canonical response of GH during OGTT. In panel (C) the bars heights correspond to the number of patients with a paradoxical increase of GH observed that begin at the time point indicated in the $x$-axis during OGTT.

and GIPR-L adenomas respectively (Fig. 5A, Pearson $\chi^{2}$ $13.75, P<0.001)$. In contrast, a frequency of $\sim 40 \%$ among paradoxical acromegalic patients could be observed in $g s p^{+}$(4/9) and $g s p^{-}$(16/38) (Fig. 5B).

Although patients with GIPR-H tumors tended to be males (50\% of males vs $27 \%$ of females, $P=0.18$ ) with higher serum GH levels $(26.8 \pm 29.4$ vs $17.2 \pm 15.9$, $P=0.159)$, these differences were not statistically significant. In addition, no differences were observed in the age at diagnosis, basal plasma PRL levels or in the distribution of macro and micro adenomas between GIPR-H and GIPR-L groups. We then evaluated the initial $\mathrm{GH}$ increase in patients with GH-PI according to the somatotropinoma GIPR expression status. In 77\% (10/13) of patients with a GIPR-H tumor, GH increase occurred very early during OGTT (i.e. within $30 \mathrm{~min}$ ), whereas only in one-third of patients with a GIPR-L adenoma $(2 / 7)$ $(P=0.06$, Fig. 5C).

\section{Cell lines, transfection, dual-luciferase assay}

To test if the high levels of circulating GIP reported for acromegalic patients (11) might be a direct consequence of high GH or IGF-1, GIP promoter inducibility was assessed in the murine enteroendocrine cell line STC-1. hGIP2.9kb-luc-transfected STC-1 cells were treated with increasing concentrations of either IGF-1 or GH, and luciferase activity was determined. A $100 \mathrm{nM}$ insulin treatment was used as positive control.

As reported in Fig. 6A, the IGF-1 treatment induced a dose-dependent increase in luciferase activity with a significant effect already at $0.1 \mathrm{ng} / \mathrm{mL}(P<0.05)$. The maximum increase $(6.4$-fold $\pm 0.5, P>0.001)$ was reached at $50 \mathrm{ng} / \mathrm{mL}$, comparable with that generated by IBMX + FK used as second positive control of GIP promoter activation. In contrast, no induction of the GIP promoter activity was seen at any concentration of $\mathrm{GH}$. These data are consistent

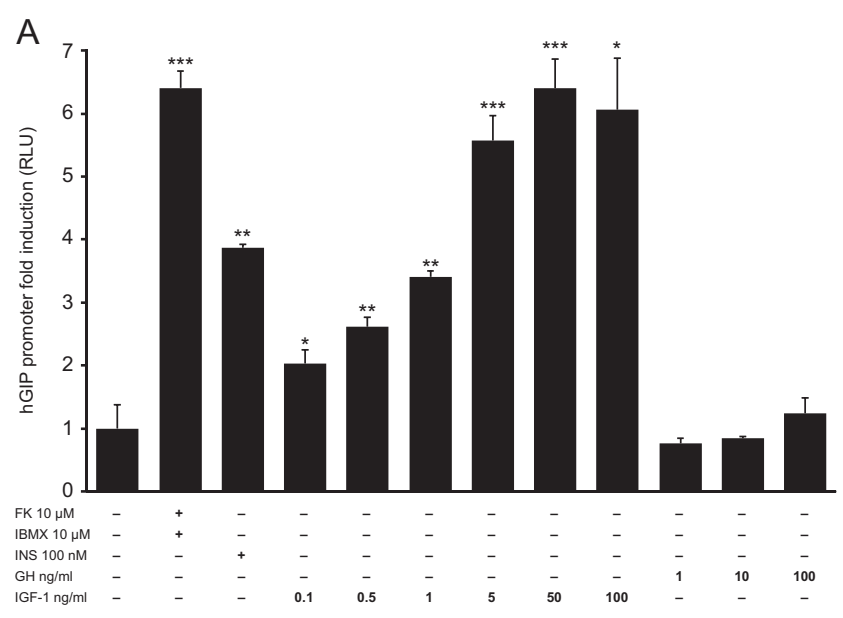

B

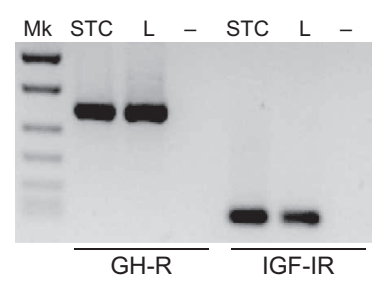

Figure 6

Effect of GH, IGF-1 and insulin on GIP promoter activity in STC-1 cells. (A) STC-1 cells were transfected with the GIP-luc reporter plasmid (hGIP2.9kb-luc), and luciferase expression was measured in the presence of increasing concentrations of GH or IGF-1 or of insulin $(100 \mathrm{nM})$ or a combination of IBMX + FK (the two latter are positive control of GIP-luc induction). The relative activity was adjusted for transfection efficiency using pRL-TK. Results are expressed as relative luciferase activity compared with basal level of GIP-luc-transfected STC-1 without treatments. Error bars represent standard deviation of the mean. ${ }^{*} P<0.05, * * P<0.01$, $\star * * P<0.001$, compared with cells given no drug treatment (t-test). All experiments were performed at least in triplicate. (B) IGF-1 and GH receptor expression in STC-1 cells (STC). L mouse liver, Mk marker, - negative control. 
with the observation that IGF-1R is expressed in STC-1 cells (Fig. 6B).

\section{Discussion}

Recently two studies - one by our group - raised the hypothesis that GIP/GIPR could promote the GH-PI in a proportion of acromegalic patients $(3,10)$ by counteracting hypothalamic inhibitory activity induced by glucose $(19,20)$. In the current study, we confirmed that impaired expression of a functional GIPR, rather than an altered hypothalamic somatostatinergic activity (21), mainly contributes to this effect. Indeed, previous observations of functional coupling of GIPR with Gos in GIPR-transfected GH3 cells (3), and the current findings of GIP responsiveness of most high GIPR-expressing primary cultures, strongly suggest that in about 30\% of GH-sec PAs, activation of an unconventional GIP/GIPR axis, after an oral glucose administration, stimulates $\mathrm{GH}$ secretion by mimicking the cellular events triggered by GHRH stimulation $(22,23,24)$. The co-localization of GIPR and GH in somatotroph tumor cells further supports the assumption of a mechanism of action that parallels the GIP-mediated cortisol synthesis and secretion in FDCS patients (8).

In nearly $20 \%$ of cases, however, GH plasma levels increase after glucose stimuli in spite of low GIPR levels and vice versa - i.e., high GIPR level without GH-PI. Given the high specificity that characterizes the GIP/GIPR binding (25), in the former cases, GH-PI is unlikely caused by GIP binding to alternative GPCRs, which could instead be directly activated by a gastroenteropancreatic hormone (e.g. glucagon-like peptide-1). The secondary activation by an hypothalamus-mediated mechanism after an hyperglycemic stimulus (e.g. GnRH (26)) may represent an alternative hypothesis to explain this phenomenon, being a functional GnRH receptor identified in nearly $15 \%$ of $g s p^{-}$GH-sec PA (27). Looking to paradoxical cases, the evidence that in GIPR-negative patients GH increase occurs later during the test compared to GIPRpositive suggests, in addition, that a GPCR-independent mechanism cannot be completely ruled out and that the GH-PI may occur when hyperglycemia cannot abolish the endogenous GHRH-induced GH rise, a mechanism proposed for explaining the GH-PI observed in $8 \%$ of normal subjects (34). On the other hand, neither GIPRinactivating mutations nor nucleotide variants (i.e. SNP rs1800437) associated to enhanced receptor desensitization (28) explain GIP unresponsiveness in
GIPR-H cases. Although technical reasons could not be fully ruled out, mechanisms affecting GPCR modulation, involving $\beta$-arrestins, GPCR kinases or regulators of G-protein signaling, as already associated to SSTRs activation (29), may represent an intriguing alternative.

Besides an higher incidence of GH-PI, as in FDCS (30) the presence of GIPR overexpression does apparently not influence the major clinical features in acromegalic patients as already reported in a subset of the present cohort (3). This partially reflects the results of previous studies in which patients' clinical features have been analyzed with respect to gsp mutational status. Besides a slightly higher responsiveness to SA (31) and a more common small size and densely granulated pattern reported for $g s p^{+}$tumors, no relevant difference in clinical features (e.g. GH/IGF-1 levels, disease duration, cure rate and outcome) have been found $(32,33)$. This may imply that although the improper cAMP pathway activation represents a compelling mechanism for explaining $\mathrm{GH}$ cell hypersecretion, its clinical significance has not been clearly defined.

GIPR-overexpressing $\mathrm{GH}$-sec PAs respond more frequently and effectively to the adenylate cyclase activator FK by increasing GH secretion, in accordance with previous observations of a less inducible cAMPsignaling pathway in $g s p^{+}$tumors (18). The exclusion of these latter from our cohort to specifically determine the sole contribution of GIPR to this, however, greatly reduces the cohort size and prevents us from drawing final conclusions on this aspect, despite a tendency toward a higher responsiveness to FK in GIPR-H compared to GIPR-L/ $g s p^{-}$emerges. Evaluation of a larger cohort would be desirable to allow a reliable subgroup analysis. Given the correlation between GIPR overexpression and GH-PI, a possible relationship between GIPR mRNA steady-state level and clinical features could be indirectly inferred, thus stratifying acromegalic patients according to OGTT response. To our knowledge, no studies investigating the possibility of such an association have been performed, except for one showing that the simultaneous presence of a GH paradoxical response to TRH and the lack of partial GH inhibition after OGTT were related to smaller tumor size and postoperative long-term control of the disease (21).

Activating mutations in the gene promoter (15) or transcribed regions are unlikely to underlie GIPR overexpression in GH-sec PAs. This resembles FDCS tumors $(34,35)$ that does not even present changes in the level of transcription factor - e.g. Sp1/Sp3, Pax6 and Foxa1/Foxa2 $(34,36,37)$ - involved in GIPR expression 
regulation $(38,39,40)$. Based on the quantification of flanking gene expression and GIPR copy number, we can also exclude a general (40) or locus-specific impairment of transcription as well as of a gross chromosomal duplication. A recent large integrated epigenomic and transcriptomic study associated a GIPR locus methylation defect to gene expression impairment (41). Although this mechanism seems at the moment the most probable, alternative genetic events - e.g promoter swapping, gene fusion - need to be considered for definitely explaining this phenomenon.

Regardless of the reasons for this upregulation, these data together suggest that the aberrant expression of GIPR may be a secondary rather than a primary event. This has been recently reported in Cushing's disease and aldosteronomas in which EGFR or GnRHR overexpression is secondary to USP8 deubiquitinase gene mutations (42) or to b-catenin constitutive active mutations (43) respectively. On the other hand, the observation that GIPR-altered expression may be sufficient to trigger adrenocortical tumor formation (7) and its mutual exclusivity with ARMC5 gene mutations in patients with bilateral macronodular adrenal hyperplasia $(16,44)$ cannot exclude the possibility that GIPR overexpression might be the pivotal driver of tumor transformation in endocrine tissues.

In a previous report, in spite of the recognized analogies in the mechanism regulating the aberrant secretion of GH and cortisol in GIPR-expressing GH-sec PA and adrenal nodules respectively, aberrant activation of the GIP/GIPR pathway has been excluded as the primary cause of acromegaly as the GH blood concentration remains always above the diagnostic threshold (10). The abnormally high fasting and postprandial plasma GIP levels in acromegalic patients (11) led us to postulate that in GIPR-expressing PAs, GIP plasma levels could be high enough to chronically trigger adenylyl cyclase/ cAMP signaling (3). In addition, the stimulatory effect of IGF-1 on GIPR promoter activity in STC-1 may suggest the possible presence of a self-sustaining GH/IGF-I/GIP axis in these patients. Food dependency indeed might characterize the first phase of the disease and prolonged exposure to high circulating GH levels might induce persistently elevated GIP levels that continuously trigger the adenylyl cyclase/cAMP signaling cascade in the GIPRoverexpressing GH-sec PA. Higher basal circulating GH levels should characterize a persistent cAMP stimulation - i.e. patients overexpressing GIPR - which we have seen only as tendency. On the other hand, a similar expectation could also be formulated for $g s p^{+}$samples for which, on the contrary, data are conflicting and inconclusive (33). This may imply that although important in tumoral transformation, as confirmed by recent NGS studies (45), an impaired cAMP pathway could not be used to predict basal serum GH levels.

In conclusion, we here demonstrate for the first time that in GH-sec PAs, the functional GIP/GIPR pathway mediates GH secretion and is frequently associated with GH-PI. Whether GIPR overexpression may be associated with a specific clinical or molecular phenotype in GH-sec PAs and acromegaly, and may thus be explored for novel therapeutic approaches, as recently proposed for neuroendocrine tumors (46), remains to be established. Finally, further studies based on a large number of cases and the development of a stable transgenic model with inducible GIPR overexpression targeted to pituitary somatotroph lineage will be required to establish the real role of GIPR overexpression in the pathogenesis of $\mathrm{GH}-\mathrm{sec}$ PAs and to establish the possible existence of a GH/IGF-1/GIP axis in a proportion of acromegalic patients.

\section{Supplementary data}

This is linked to the online version of the paper at http://dx.doi.org/10.1530/ EJE-16-0831.

Declaration of interest

The authors declare that there is no conflict of interest that could be perceived as prejudicing the impartiality of this study.

\section{Funding}

This research did not receive any specific grant from any funding agency in the public, commercial or not-for-profit sector.

\section{Acknowledgments}

The authors are grateful to Prof John Funder for copy-editing the manuscript.

\section{References}

1 Chanson P, Salenave S, Kamenicky P, Cazabat L \& Young J. Pituitary tumours: acromegaly. Best Practice and Research Clinical Endocrinology and Metabolism 200923 555-574. (doi:10.1016/j.beem.2009.05.010)

2 Melmed S. Acromegaly pathogenesis and treatment. Journal of Clinical Investigation 2009119 3189-3202. (doi:10.1172/JCI39375)

3 Occhi G, Losa M, Albiger N, Trivellin G, Regazzo D, Scanarini M, Monteserin-Garcia JL, Frohlich B, Ferasin S, Terreni MR et al. The glucose-dependent insulinotropic polypeptide receptor is overexpressed amongst GNAS1 mutation-negative somatotropinomas and drives growth hormone (GH)-promoter activity in GH3 cells. Journal of Neuroendocrinology 201123 641-649. (doi:10.1111/j.13652826.2011.02155.x)

4 Volz A, Goke R, Lankat-Buttgereit B, Fehmann HC, Bode HP \& Goke B. Molecular cloning, functional expression, and signal 
transduction of the GIP-receptor cloned from a human insulinoma. FEBS Letters 1995373 23-29. (doi:10.1016/0014-5793(95)01006-Z)

5 Gremlich S, Porret A, Hani EH, Cherif D, Vionnet N, Froguel P $\&$ Thorens B. Cloning, functional expression, and chromosomal localization of the human pancreatic islet glucose-dependent insulinotropic polypeptide receptor. Diabetes 199544 1202-1208. (doi:10.2337/diab.44.10.1202)

6 Seino Y, Fukushima M \& Yabe D. GIP and GLP-1, the two incretin hormones: similarities and differences. Journal of Diabetes Investigation 20101 8-23. (doi:10.1111/j.2040-1124.2010.00022.x)

7 Mazzuco TL, Chabre O, Feige JJ \& Thomas M. Aberrant GPCR expression is a sufficient genetic event to trigger adrenocortical tumorigenesis. Molecular and Cellular Endocrinology 2007 265-266 23-28. (doi:10.1016/j.mce.2006.12.034)

8 Lacroix A, Baldacchino V, Bourdeau I, Hamet P \& Tremblay J. Cushing's syndrome variants secondary to aberrant hormone receptors. Trends in Endocrinology and Metabolism 200415 375-382. (doi:10.1016/S1043-2760(04)00188-2)

9 Albiger NM, Occhi G, Mariniello B, Iacobone M, Favia G, Fassina A, Faggian D, Mantero F \& Scaroni C. Food-dependent Cushing's syndrome: from molecular characterization to therapeutical results. European Journal of Endocrinology 2007157 771-778. (doi:10.1530/EJE07-0253)

10 Umahara M, Okada S, Ohshima K \& Mori M. Glucose-dependent insulinotropic polypeptide induced growth hormone secretion in acromegaly. Endocrine Journal 200350 643-650. (doi:10.1507/ endocri.50.643)

11 Peracchi M, Porretti S, Gebbia C, Pagliari C, Bucciarelli P, Epaminonda P, Manenti S \& Arosio M. Increased glucose-dependent insulinotropic polypeptide (GIP) secretion in acromegaly. European Journal of Endocrinology 2001145 R1-R4. (doi:10.1530/eje.0.145R001)

12 Katznelson L, Laws ER Jr, Melmed S, Molitch ME, Murad MH, Utz A, Wass JA \& Endocrine Society. Acromegaly: an endocrine society clinical practice guideline. Journal of Clinical Endocrinology and Metabolism 201499 3933-3951. (doi:10.1210/jc.2014-2700)

13 Occhi G, Regazzo D, Albiger NM, Ceccato F, Ferasin S, Scanarini M, Denaro L, Cosma C, Plebani M, Cassarino MF et al. Activation of the dopamine receptor type-2 (DRD2) promoter by 9-cis retinoic acid in a cellular model of Cushing's disease mediates the inhibition of cell proliferation and ACTH secretion without a complete corticotroph-tomelanotroph transdifferentiation. Endocrinology 2014155 3538-3549. (doi:10.1210/en.2013-1820)

14 Occhi G, Albiger N, Berlucchi S, Gardiman M, Scanarini M, Scienza R, Fassina A, Mantero F \& Scaroni C. Peroxisome proliferator-activated receptor gamma in the human pituitary gland: expression and splicing pattern in adenomas versus normal pituitary. Journal of Neuroendocrinology 200719 552-559. (doi:10.1111/j.13652826.2007.01562.x)

15 Boylan MO, Jepeal LI \& Wolfe MM. Sp1/Sp3 binding is associated with cell-specific expression of the glucose-dependent insulinotropic polypeptide receptor gene. American Journal of Physiology: Endocrinology and Metabolism 2006290 E1287-E1295. (doi:10.1152/ ajpendo.00535.2005)

16 Albiger NM, Regazzo D, Rubin B, Ferrara AM, Rizzati S, Taschin E, Ceccato F, Arnaldi G, Pecori Giraldi F et al. A multicenter experience on the prevalence of ARMC5 mutations in patients with primary bilateral macronodular adrenal hyperplasia: from genetic characterization to clinical phenotype. Endocrine 201655 959-968. (doi:10.1007/s12020-016-0956-z)

17 Fujita Y, Chui JW, King DS, Zhang T, Seufert J, Pownall S, Cheung AT $\&$ Kieffer TJ. Pax6 and Pdx1 are required for production of glucosedependent insulinotropic polypeptide in proglucagon-expressing L cells. American Journal of Physiology: Endocrinology and Metabolism 2008 295 E648-E657. (doi:10.1152/ajpendo.90440.2008)
18 Landis CA, Masters SB, Spada A, Pace AM, Bourne HR \& Vallar L. GTPase inhibiting mutations activate the alpha chain of Gs and stimulate adenylyl cyclase in human pituitary tumours. Nature 1989 340 692-696. (doi:10.1038/340692a0)

19 Penalva A, Burguera B, Casabiell X, Tresguerres JA, Dieguez C \& Casanueva FF. Activation of cholinergic neurotransmission by pyridostigmine reverses the inhibitory effect of hyperglycemia on growth hormone $(\mathrm{GH})$ releasing hormone-induced GH secretion in man: does acute hyperglycemia act through hypothalamic release of somatostatin? Neuroendocrinology 198949 551-554. (doi:10.1159/000125166)

20 Balzano S, Loche S, Murtas ML, Fanni T, Sica V, Pintor C \& Martino E. Potentiation of cholinergic tone counteracts the suppressive effect of oral glucose administration on the GH response to GHRH in man. Hormone and Metabolic Research 198921 52-53. (doi:10.1055/s-2007-1009149)

21 De Marinis L, Mancini A, Bianchi A, Gentilella R, Valle D, Giampietro A, Zuppi P, Anile C, Maira G \& Giustina A. Preoperative growth hormone response to thyrotropin-releasing hormone and oral glucose tolerance test in acromegaly: a retrospective evaluation of 50 patients. Metabolism: Clinical and Experimental 200251 616-621. (doi:10.1053/meta.2002.32017)

22 Bilezikjian LM \& Vale WW. Stimulation of adenosine $3^{\prime}, 5^{\prime}$-monophosphate production by growth hormone-releasing factor and its inhibition by somatostatin in anterior pituitary cells in vitro. Endocrinology 1983113 1726-1731. (doi:10.1210/endo-113-5-1726)

23 Bilezikjian LM, Erlichman J, Fleischer N \& Vale WW. Differential activation of type I and type II 3',5'-cyclic adenosine monophosphatedependent protein kinases by growth hormone-releasing factor. Molecular Endocrinology 1987 137-146. (doi:10.1210/mend-1-2-137)

24 Shepard AR, Zhang W \& Eberhardt NL. Two CGTCA motifs and a GHF1/Pit1 binding site mediate cAMP-dependent protein kinase A regulation of human growth hormone gene expression in rat anterior pituitary GC cells. Journal of Biological Chemistry 1994269 1804-1814.

25 Moon MJ, Kim HY, Kim SG, Park J, Choi DS, Hwang JI \& Seong JY. Tyr1 and Ile7 of glucose-dependent insulinotropic polypeptide (GIP) confer differential ligand selectivity toward GIP and glucagonlike peptide-1 receptors. Molecules and Cells 201030 149-154. (doi:10.1007/s10059-010-0100-5)

26 Roland AV \& Moenter SM. Regulation of gonadotropin-releasing hormone neurons by glucose. Trends in Endocrinology and Metabolism 201122 443-449. (doi:10.1016/j.tem.2011.07.001)

27 Lania A, Mantovani G, Ferrante E, Zavanone LM, Locatelli M, Corbetta S, Beck-Peccoz P \& Spada A. Gonadotropin-releasing hormone initiates multiple signaling pathways in human GH-secreting adenomas. Journal of Endocrinological Investigation 2004 27 328-333. (doi:10.1007/BF03351057)

28 Mohammad S, Patel RT, Bruno J, Panhwar MS, Wen J \& McGraw TE. A naturally occurring GIP receptor variant undergoes enhanced agonist-induced desensitization, which impairs GIP control of adipose insulin sensitivity. Molecular and Cellular Biology 201434 3618-3629. (doi:10.1128/MCB.00256-14)

29 Gatto F, Feelders R, van der Pas R, Kros JM, Dogan F, van Koetsveld PM, van der Lelij AJ, Neggers SJ, Minuto F, de Herder $\mathrm{W}$ et al. beta-Arrestin 1 and 2 and $\mathrm{G}$ protein-coupled receptor kinase 2 expression in pituitary adenomas: role in the regulation of response to somatostatin analogue treatment in patients with acromegaly. Endocrinology 2013154 4715-4725. (doi:10.1210/ en.2013-1672)

$30 \mathrm{El}$ Ghorayeb N, Bourdeau I \& Lacroix A. Multiple aberrant hormone receptors in Cushing's syndrome. European Journal of Endocrinology 2015173 M45-M60. (doi:10.1530/EJE-15-0200)

31 Efstathiadou ZA, Bargiota A, Chrisoulidou A, Kanakis G, Papanastasiou L, Theodoropoulou A, Tigas SK, Vassiliadi DA, Alevizaki M \& Tsagarakis S. Impact of gsp mutations in somatotroph 
pituitary adenomas on growth hormone response to somatostatin analogs: a meta-analysis. Pituitary 201518 861-867. (doi:10.1007/ s11102-015-0662-5)

32 Spada A, Arosio M, Bochicchio D, Bazzoni N, Vallar L, Bassetti M \& Faglia G. Clinical, biochemical, and morphological correlates in patients bearing growth hormone-secreting pituitary tumors with or without constitutively active adenylyl cyclase. Journal of Clinical Endocrinology and Metabolism 199071 1421-1426. (doi:10.1210/jcem71-6-1421)

33 Buchfelder M, Fahlbusch R, Merz T, Symowski H \& Adams EF. Clinical correlates in acromegalic patients with pituitary tumors expressing GSP oncogenes. Pituitary 19991 181-185. (doi:10.102 3/A:1009905131334)

34 Antonini SR, N’Diaye N, Baldacchino V, Hamet P, Tremblay J \& Lacroix A. Analysis of the putative regulatory region of the gastric inhibitory polypeptide receptor gene in food-dependent Cushing's syndrome. Journal of Steroid Biochemistry and Molecular Biology 200491 171-177. (doi:10.1016/j.jsbmb.2004.03.120)

35 N’Diaye N, Tremblay J, Hamet P, De Herder WW \& Lacroix A. Adrenocortical overexpression of gastric inhibitory polypeptide receptor underlies food-dependent Cushing's syndrome. Journal of Clinical Endocrinology and Metabolism $1998 \mathbf{8 3}$ 2781-2785. (doi:10.1210/jc.83.8.2781)

36 Gosmain Y, Cheyssac C, Masson MH, Guerardel A, Poisson C \& Philippe J. Pax6 is a key component of regulated glucagon secretion. Endocrinology 2012153 4204-4215. (doi:10.1210/en.2012-1425)

37 Heddad Masson M, Poisson C, Guerardel A, Mamin A, Philippe J \& Gosmain Y. Foxa1 and Foxa2 regulate alpha-cell differentiation, glucagon biosynthesis, and secretion. Endocrinology 2014155 3781-3792. (doi:10.1210/en.2013-1843)

38 Bourdeau I, Antonini SR, Lacroix A, Kirschner LS, Matyakhina L, Lorang D, Libutti SK \& Stratakis CA. Gene array analysis of macronodular adrenal hyperplasia confirms clinical heterogeneity and identifies several candidate genes as molecular mediators. Oncogene 200423 1575-1585. (doi:10.1038/sj.onc.1207277)

39 Baldacchino V, Oble S, Decarie PO, Bourdeau I, Hamet P, Tremblay J $\&$ Lacroix A. The Sp transcription factors are involved in the cellular expression of the human glucose-dependent insulinotropic polypeptide receptor gene and overexpressed in adrenals of patients with Cushing's syndrome. Journal of Molecular Endocrinology 200535 61-71. (doi:10.1677/jme.1.01765)

40 Lampron A, Bourdeau I, Hamet P, Tremblay J \& Lacroix A. Whole genome expression profiling of glucose-dependent insulinotropic peptide (GIP)- and adrenocorticotropin-dependent adrenal hyperplasias reveals novel targets for the study of GIP-dependent Cushing's syndrome. Journal of Clinical Endocrinology and Metabolism 200691 3611-3618. (doi:10.1210/jc.2006-0221)

41 Karpathakis A, Dibra H, Pipinikas C, Feber A, Morris T, Francis J, Oukrif D, Mandair D, Pericleous M, Mohmaduvesh M et al. Prognostic impact of novel molecular subtypes of small intestinal neuroendocrine tumor. Clinical Cancer Research 201622 250-258. (doi:10.1158/1078-0432.CCR-15-0373)

42 Reincke M, Sbiera S, Hayakawa A, Theodoropoulou M, Osswald A, Beuschlein F, Meitinger T, Mizuno-Yamasaki E, Kawaguchi K, Saeki Y et al. Mutations in the deubiquitinase gene USP8 cause Cushing's disease. Nature Genetics 201547 31-38. (doi:10.1038/ ng.3166)

43 Teo AE, Garg S, Shaikh LH, Zhou J, Karet Frankl FE, Gurnell M, Happerfield L, Marker A, Bienz M, Azizan EA et al. Pregnancy, primary aldosteronism, and adrenal CTNNB1 mutations. New England Journal of Medicine 2015373 1429-1436. (doi:10.1056/ NEJMoa1504869)

44 Espiard S, Drougat L, Libe R, Assie G, Perlemoine K, Guignat L, Barrande G, Brucker-Davis F, Doullay F, Lopez S et al. ARMC5 Mutations in a large cohort of primary macronodular adrenal hyperplasia: clinical and functional consequences. Journal of Clinical Endocrinology and Metabolism 2015100 E926-E935. (doi:10.1210/ jc.2014-4204)

45 Ronchi CL, Peverelli E, Herterich S, Weigand I, Mantovani G, Schwarzmayr T, Sbiera S, Allolio B, Honegger J, Appenzeller S et al. Landscape of somatic mutations in sporadic GH-secreting pituitary adenomas. European Journal of Endocrinology/European Federation of Endocrine Societies 2016174 363-372. (doi:10.1530/EJE-15-1064)

46 Sherman SK, Carr JC, Wang D, O’Dorisio MS, O’Dorisio TM \& Howe JR. Gastric inhibitory polypeptide receptor (GIPR) is a promising target for imaging and therapy in neuroendocrine tumors. Surgery 2013154 1206-1213; discussion 1214. (doi:10.1016/j. surg.2013.04.052)

Received 4 October 2016

Revised version received 1 February 2017

Accepted 7 February 2017 